\title{
Stimulated-emission-induced enhancement of the decay rate of longitudinal optical phonons in III-V semiconductors
}

\author{
Jing Chen ${ }^{\text {a) }}$ and Jacob B. Khurgin ${ }^{\text {b) }}$ \\ Department of Electrical and Computer Engineering, Johns Hopkins University, Baltimore, Maryland 21218 \\ R. Merlin ${ }^{\mathrm{c})}$ \\ Department of Physics, University of Michigan, Ann Arbor, Michigan 48109-1120
}

(Received 18 December 2001; accepted for publication 20 February 2002)

\begin{abstract}
We explore the feasibility of reducing the lifetime of longitudinal optical phonons in InP by injecting coherent longitudinal acoustic modes of frequency given by the difference between those of the longitudinal and transverse optical phonons. Calculations show that a ten-fold reduction in the lifetime can be attained using a $40 \mathrm{~mW}$ acoustic source. The increase in the phonon decay should reduce the scattering rate of hot electrons in fast devices. (c) 2002 American Institute of Physics.
\end{abstract}

[DOI: $10.1063 / 1.1471567$ ]

In high-speed electronic and optoelectronic devices based on polar III-V semiconductors, the performance is ultimately limited by the scattering of carriers by phonons. ${ }^{1,2}$ Due to the dominance of the Fröhlich interaction, and its long-range nature which favors long wavelength, the carriers interact primarily with LO phonons of wave vector $q \sim 0$. The scattering rates increase with increasing LO density and, in the absence of a mechanism to remove these modes, the electron mobility is drastically reduced leading to a deterioration of the device performance, particularly for the speed and power consumption. Since the phonons produced by the scattering of the electrons are LO phonons close to the zone center and, thus, with near-zero group velocity, removal by diffusion is very slow. Hence the only process preventing the LO phonon from buildup is their decay into various combinations of lower frequency modes ${ }^{3}$ which interact weakly with carriers. Unfortunately, in many semiconductors LO phonons live quite a long life, mostly due to the low joint (two-phonon) density of final TO, TA, or LA states into which LO phonons decay. This applies in particular to the case of InP where the LO phonon lifetime is $\sim 200 \mathrm{ps}^{4}$ and the dominant decay channel is $\mathrm{LO} \rightarrow \mathrm{TO}+\mathrm{LTA}^{3}{ }^{3,4}$

In this letter we explore the possibility of enhancing the LO phonon decay rate in InP by injecting coherent acoustic phonons of the proper frequency to induce stimulated emission. In some sense, what we propose to do is to create a parametric amplifier of acoustic phonons where, by analogy with the optical counterpart, the zone-center optical modes would act as the "pump," the externally injected LA phonons as the "signal" and the TO mode as the "idler." Within this context, we note that the population of the signal LA phonons is expected to be amplified as a byproduct of the stimulated process.

The possibility that stimulated emission may shorten the lifetime of the LO phonons in polar semiconductors was first considered in Ref. 5. This work shows that for GaAs, under

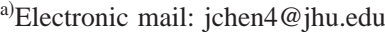

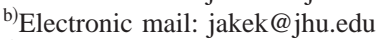

${ }^{c)}$ Electronic mail: merlin@umich.edu nonequilibrium conditions, the $\mathrm{LO} \rightarrow \mathrm{LA}+\mathrm{LA}$ process becomes dominant in the presence of a large density of LA modes. To attain occupation numbers for the "daughter" LA phonons close to unity, the phonon generation rate needs to be as large as $10^{24} \mathrm{~cm}^{-3} \mathrm{~s}^{-1}$, which is quite high. In Ref. 5, only the Klemens $\mathrm{LO} \rightarrow \mathrm{LA}+\mathrm{LA}$ channel $^{6}$ was considered even though, according to Ref. 3, 95\% of the decay is due to $\mathrm{LO} \rightarrow \mathrm{LA}+\mathrm{TA}$ processes.

Here, we propose a scheme in which stimulated emission is the result of externally injected coherent LA phonons of relatively low $(\sim 1 \mathrm{THz})$ frequency. We predict that a substantial (more than tenfold) reduction in the effective LO lifetime can be achieved at relatively low LA densities. Our approach is illustrated in Fig. 1 where the LA phonons are generated in the region on the left. It has been shown that coherent LA modes can be generated optically, via transient stimulated Brillouin scattering or using stimulated Raman scattering by folded acoustic modes in superlattices. ${ }^{7-10} \mathrm{We}$ also note that the possibility that phonon scattering by intersubband transitions in quantum wells may lead to coherent emission as discussed in Ref. 11.

In our mechanism, the injected LA phonons enter the active region of the device where hot carriers are present,

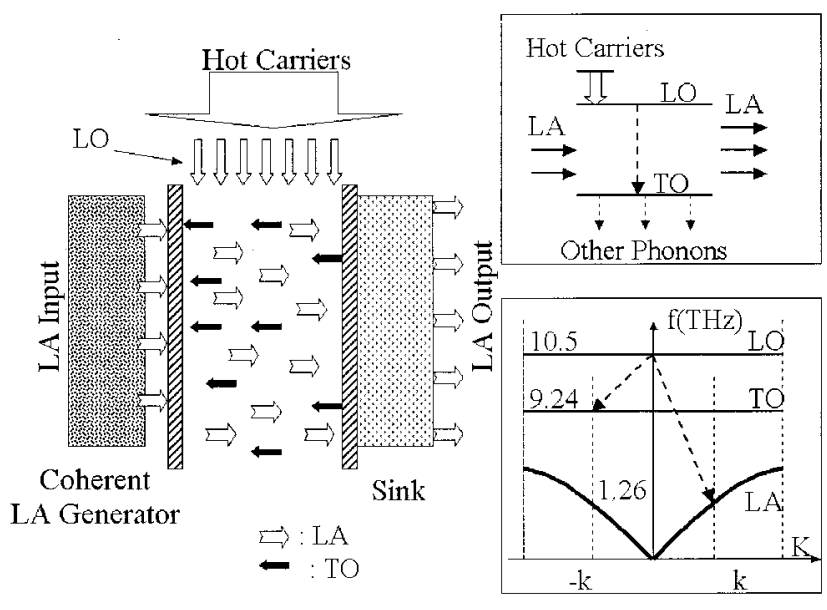

FIG. 1. Stimulated phonon cooling (energy diagram in the inset). 
TABLE I. Parameters for InP.

\begin{tabular}{lccc}
\hline \hline & LO & LA & TO \\
\hline Group velocity $\left(10^{5} \mathrm{~cm} / \mathrm{s}\right)$ & & 4.5 & \\
Frequency $(\mathrm{THz})$ & 10.5 & 1.26 & 9.24 \\
Spontaneous lifetime $(\mathrm{ps})$ & $204^{4}$ & $6000^{16}$ & $10.6^{15}$ \\
\hline \hline
\end{tabular}

typically a channel of a field-effect transistor where LO phonons are generated. As mentioned earlier, the main decay channel in InP is LO $\rightarrow$ TO + LTA. ${ }^{3}$ Thus, if the energy and momentum of the LA phonons is properly chosen, stimulated LO decay will follow. The LA modes continue to propagate towards the sink on the right-hand side, while the TO phonons decay into various combinations of LA and TA phonons. Since the (deformation potential) interaction of these modes with carriers is relatively weak, the electron transport behavior will not be affected much by the associated increase in the density of TO phonons.

Using the optical analogy and the fact that phonons are bosons, the proposed scheme can be described as an "acoustic parametric amplifier." Since the group velocities of LO and TO phonons are close to zero, they can be loosely considered to be the upper and lower states of a LA phonon amplifier, and the whole scheme then bears a close relationship to laser cooling.

We also note that, if proper feedback is provided, our scheme leads to a phonon laser. Our proposal involving only phonon states is significantly different from other schemes ${ }^{12,13}$ which rely on sharp resonant transition between two electronic states.

Let us now estimate the LA phonon density and, correspondingly, the strain necessary for an appreciable reduction in the LO phonon lifetime. The relevant parameters for InP are shown in Table I. Consider the general expression for the decay rate of LO phonons of frequency $\omega_{0}$ (LO), whose occupation number is $n_{0}$ into a pair of lower-energy phonons with frequencies $\omega_{1}(\mathrm{LA})$ and $\omega_{2}(\mathrm{TO})::^{3,5,14}$

$$
\begin{aligned}
R= & \sum_{k_{1}, k_{2}} \pi h^{2} U_{a}^{2}\left(4 N m^{3} \omega_{0} \omega_{1} \omega_{2}\right)^{-1} \delta\left(h \omega_{0}-h \omega_{1}-h \omega_{2}\right) \\
& \times \delta_{0, k_{1}+k_{2}}\left[n_{0}\left(n_{1}+1\right)\left(n_{2}+1\right)-\left(n_{0}+1\right) n_{1} n_{2}\right],
\end{aligned}
$$

where $U_{a}$ is the strain energy density, $N$ is number of unit cells, and $m$ is the average mass of the atoms. Introducing the density of phonons, $N_{i}=n_{i} / V$, the matrix element for the interaction $M^{2}=\pi \hbar^{2} U_{a}^{2} V\left(32 N m^{3} \pi^{3} f_{0} f_{1} f_{2}\right)^{-1}$, and the two-phonon density of states $g\left(h \omega_{0}\right)=V^{-1} \sum_{k_{1}, k_{2}}$ $\times \delta_{0, k_{1}+k_{2}} \delta\left(h \omega_{0}-h \omega_{1}-h \omega_{2}\right)$, we can rewrite Eq. (1) as

$$
\begin{aligned}
R= & M^{2} V N_{0} g\left(h \omega_{0}\right)+M^{2} V \sum_{k_{1} k_{2}} L\left(h \omega_{1}\right) \delta\left(h \omega_{0}-h \omega_{1}-h \omega_{2}\right) \\
& \times \delta_{0, k_{1}+k_{2}}\left(N_{0} N_{1}+N_{0} N_{2}-N_{1} N_{2}\right)=R_{\mathrm{sp}}+R_{\mathrm{st}}
\end{aligned}
$$

here, $R_{\mathrm{sp}}$ is not a function of $N_{1}$, so it stands for the spontaneous decay. And we use $L\left(\hbar \omega_{1}\right)=\Gamma_{E} \pi^{-1} /\left[\left(\hbar \bar{\omega}_{1}-\hbar \omega_{1}\right)^{2}\right.$ $\left.+\Gamma_{E}^{2}\right]$ to account for the fact that the incoming LA phonons are not monochromatic (they exhibit a distribution of frequencies around the central frequency $\bar{\omega}_{1}$ that satisfies the energy and momentum conservation conditions). Furthermore, the transition itself is broadened due to the finite life- times of the LO and TO phonons. Since the lifetime of the TO phonons in InP is by far the shortest, $\tau_{\mathrm{TO}} \sim 10 \mathrm{ps},{ }^{15}$ it is a good approximation to assume that $\Gamma_{E} \sim \hbar / \tau_{\mathrm{TO}}$. Performing the summation in Eq. (2), we obtain for the rate of stimulated emission

$$
\begin{aligned}
R_{\mathrm{st}} & =M^{2} V \frac{\left(N_{0} N_{1}+N_{0} N_{2}-N_{1} N_{2}\right)}{\pi \Gamma_{E}} \\
& =R_{\mathrm{sp}} \frac{\left(N_{0} N_{1}+N_{0} N_{2}-N_{1} N_{2}\right)}{N_{0} N_{t h}},
\end{aligned}
$$

where we have introduced a threshold density of injected phonons, $N_{\text {th }}=g\left(\hbar \omega_{0}\right) \pi \Gamma_{E}$. The physical meaning of this parameter is clear. $N_{\text {th }}$ is the volume density of states whose energies are within the linewidth of the externally injected LA phonons. It follows that stimulated decay overcomes spontaneous decay when the density of injected coherent phonons $N_{1}$ exceeds that of $N_{\text {th }}$.

A rough estimate of $N_{\text {th }}$ for InP can be obtained as follows. Assuming the Debye approximation, we get for 1.26 $\mathrm{THz}$ LA phonons $N_{\text {th }}=2 \pi f^{2}{ }_{\mathrm{LA}}\left(\tau_{\mathrm{TO}} \nu_{\mathrm{LA}}^{3}\right)^{-1} \approx 1.03$ $\times 10^{19} \mathrm{~cm}^{-3}$. The corresponding energy density is $8 \mathrm{~mJ} / \mathrm{cm}^{3}$ which is not exceedingly large. With this in mind, we consider the kinetic equations for the phonon densities

$$
\begin{aligned}
& \frac{\partial N_{0}}{\partial t}=G_{\mathrm{LO}}-\frac{N_{0}}{\tau_{0}}-\frac{N_{0} N_{1}+N_{0} N_{2}-N_{1} N_{2}}{\tau_{0} N_{\mathrm{th}}}, \\
& \frac{\partial N_{1}}{\partial t}+\nu_{1} \frac{\partial N_{1}}{\partial x}=\frac{N_{0} N_{1}+N_{0} N_{2}-N_{1} N_{2}}{\tau_{0} N_{\text {th }}}-\frac{N_{1}}{\tau_{1}}, \\
& \frac{\partial N_{2}}{\partial t}-\nu_{2} \frac{\partial N_{2}}{\partial x}=\frac{N_{0} N_{1}+N_{0} N_{2}-N_{1} N_{2}}{\tau_{0} N_{\text {th }}}-\frac{N_{2}}{\tau_{2}} .
\end{aligned}
$$

Here we have introduced the spontaneous LO lifetime, $\tau_{0}^{-1}=M^{2} V g\left(\hbar \omega_{0}\right)$, and their generation rate, $G_{\mathrm{LO}}$. The boundary conditions are $N_{1}(x=0) \nu_{1}=J$, where $J$ is the flux density of the injected LA phonons, and for the TO modes $N_{2}(x=L) \nu_{2}=0$. The TO phonon velocity $\nu_{2}$ in the region of interest $\left(k_{\mathrm{TO}} \sim 1.75 \times 10^{7} \mathrm{~cm}^{-1}\right)$ is $\sim 1000 \mathrm{~cm} / \mathrm{s}$, i.e., the diffusion length, $\sim 1 \AA$, is very short so that the motion of the TO phonons can be disregarded. Let us now consider the steady-state solution of Eqs. (4)-(6) under the assumption $N_{1} \gg N_{2}$, i.e., for an injected acoustic density that is much higher than that of TO phonons (we will check the validity of this assumption later). With zero group velocity for the LO and TO modes, the steady-state $(\partial N / \partial t=0)$ solutions are

$$
\begin{aligned}
& N_{2}=G_{\mathrm{LO}} \tau_{2} \frac{N_{1} \tau_{0}}{N_{\mathrm{th}} \tau_{0}+N_{1}\left(\tau_{0}+\tau_{2}\right)}, \\
& N_{0}=G_{\mathrm{LO}} \tau_{0} \frac{N_{\mathrm{th}} \tau_{0}+N_{1} \tau_{2}}{N_{\mathrm{th}} \tau_{0}+N_{1}\left(\tau_{0}+\tau_{2}\right)}=\frac{G_{\mathrm{LO}} \tau_{0}}{\alpha_{\mathrm{st}}},
\end{aligned}
$$

where $\alpha_{\mathrm{st}}$ is the enhancement factor of the LO phonon decay due to stimulated processes; its dependence on the density of externally injected LA phonons $N_{1}$ is shown in Fig. 2. One sees that, for $\tau_{0} \gg \tau_{2}$ at a high LA phonon density, the effective LO-phonon lifetime approaches asymptotically the much smaller TO-phonon lifetime. The densities of LO and TO phonons are also shown in Fig. 2. They approach each other asymptotically at high LA phonon densities. 


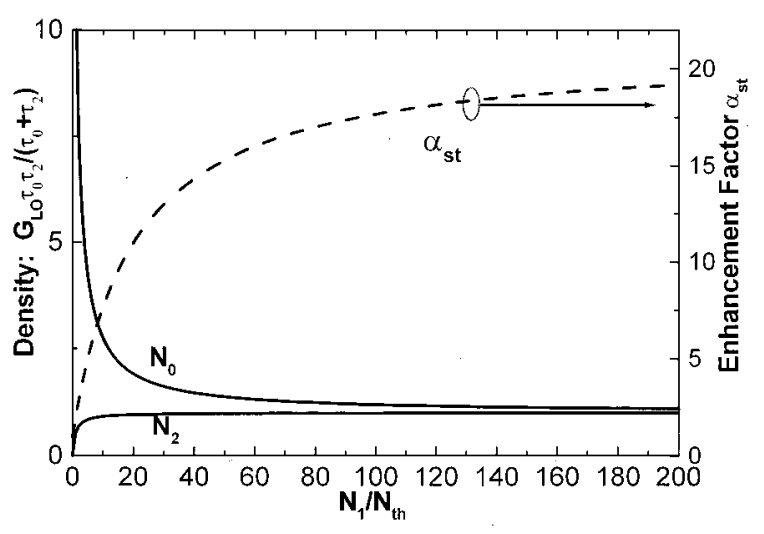

FIG. 2. $N_{0}, N_{2}$, and $\alpha_{\text {st }}$ change as functions of $N_{1} / N_{\text {th }}$ for InP.

Now, we check the validity of the assumption made earlier that the TO phonons density is always much smaller than the LA phonon density. Since we are interested in the situation when $N_{1}>10 N_{\text {th }}$, we have $N_{2} \sim G_{\mathrm{LO}} \tau_{2}<10 N_{\text {th }}$. For InP, we obtain $P_{\mathrm{LO}} \ll 10 \hbar \omega_{0} N_{\text {th }} / \tau_{2} \approx 0.7 \times 10^{11} \mathrm{~W} \mathrm{~cm}^{-3}$. For a typical electronic device with an active volume of, say, $0.2 \mu \mathrm{m} \times 1 \mu \mathrm{m} \times 100 \mu \mathrm{m}$, this results in $1 \mathrm{~W}$ power dissipation which is at least two orders of magnitude larger than that for a field-effect device. Therefore, we can disregard the TO phonon density for any practical values of the LO phonon generation rate.

To include the spatial dependence of the LA phonon density, we solve Eq. (5) under the assumptions $N_{1}>10 N_{\text {th }}$ and $\partial N_{1} / \partial t=0$ obtaining

$N_{1} \approx N_{1}(0)-N_{1}(0) \frac{x}{\nu_{1} \tau_{1}}+G_{\mathrm{LO}} \frac{x}{\nu_{1}}$.

The linearization is valid for an active region of thickness $L \sim 1 \mu \mathrm{m}$. This thickness is much smaller than the mean free path at $77 \mathrm{~K}$, which is found to be $\sim 27 \mu \mathrm{m}$ using $\tau_{\mathrm{LA}}$ $=6 \times 10^{-9} \mathrm{~s}$ at $1.26 \mathrm{THz} .{ }^{16}$

If we consider a device with $10 \mathrm{~mW}$ power consumption and active area $100 \mu \mathrm{m}^{2}$, the upper limit for the last term in Eq. (9) is $0.2 \times 10^{19} \mathrm{~cm}^{-3}$ which is 50 times smaller than $N_{1}$. Therefore, the influence of the generated LA phonons is insignificant even at very high LO generation rates. Nevertheless, it is interesting to mention that when the condition $G_{\mathrm{LO}}>N_{1}(0) / \tau_{1}$ is satisfied, there is a net amplification of the injected LA phonons as opposed to the net absorption at lower pump rates. This condition corresponds to $G_{\mathrm{LO}}$ $\sim 10^{28} \mathrm{~cm}^{-3} \mathrm{~s}^{-1}$, or $P_{\mathrm{LO}} \sim 10^{8} \mathrm{~W} \mathrm{~cm}^{-3}$ corresponding to 10 $\mathrm{mW}$ of total power. Thus, one can obtain amplification of LA phonons, but the impact of the additional LA phonons produced by stimulated emission on the LO phonon lifetime is negligibly small. This result is fundamentally different from results reported for GaAs in Ref. 5 where, in the absence of the external LA source, only a two-fold reduction in the LO lifetime was predicted at extremely high rates of LO generation. In contrast, we find here a ten-fold reduction in the lifetime that is independent of the LO generation rate.

Finally, we estimate the flux of the externally injected LA phonons needed to achieve the desired ten-fold reduction in the LO lifetime. From Fig. 2, we obtain $J=N_{1} \nu_{1} \sim 5$ $\times 10^{25} \mathrm{~cm}$, which, for a $100 \mu \mathrm{m}^{2}$ device, corresponds to a total flux of $5 \times 10^{19}$ phonons per second, or $40 \mathrm{~mW}$. We note that a substantial improvement can be achieved by using a cavity for acoustic phonons. One can also consider using a superlattice to reduce the velocity of LA phonons by zone folding. ${ }^{10}$ This would lead to an increase in the effective interaction time thereby reducing the required power. It is also of interest to consider the strain associated with the required LA density. A simple analysis shows that $N_{\mathrm{LA}} \hbar \omega_{\mathrm{LA}}$ $=\rho \omega_{\mathrm{LA}}{ }^{2} x^{2} / 2$, where $\rho$ is the density and $x$ is the atomic displacement. From this, we obtain $x \sim 0.0078 \AA$ representing a strain of $0.13 \%$. Strains of this magnitude can be obtained using ultrafast optical excitation. ${ }^{8}$

It is unlikely that our scheme can be applied to materials for which the dominant process of LO phonon decay does not involve TO phonons. When both final states of the LO decay are acoustic modes (of frequency near half that of the LO mode, i.e., in the $2-5 \mathrm{THz}$ range) the threshold density of injected LA phonons is at least a factor of 20 larger than in InP. Furthermore, the mean free path of LA phonons at 5 $\mathrm{THz}$ is rather small, $<100 \mathrm{~nm}$, making their delivery into the active region a very difficult task. Finally, if the decay mechanism involves TA phonons, we note that their lifetime is actually larger than that of LO phonons rendering the stimulated transition "self-terminating," i.e., attainable only in a pulse mode.

In conclusion, we have considered the feasibility of using stimulated emission induced by externally injected coherent LA phonons to reduce the lifetime of LO modes. For InP, we have shown that a ten-fold reduction is attainable using a $40 \mathrm{~mW}$ source of external phonons. Our results hold promise for using phonon engineering to improve the properties of electronic devices.

Work supported by the AFOSR under Contract No. F49620-00-1-0328 through the MURI program.

${ }^{1}$ W. Potz and P. Kocevar, in Hot Carriers in Semiconductor NanoStructures: Physics and Application, edited by J. Shah (Academic, Boston, 1992).

${ }^{2}$ P. G. Klemens, in Solid State Physics, edited by F. Seitz and D. Turnbull (Academic, New York, 1958), Vol. 7.

${ }^{3}$ A. Debernardi, Phys. Rev. B 57, 12847 (1998).

${ }^{4}$ F. Vallee, Phys. Rev. B 49, 2460 (1994).

${ }^{5}$ D. A. Romanov, B. A. Glavin, V. V. Mitin, and M. A. Stroscio, Phys. Rev. B 60, 4771 (1999).

${ }^{6}$ P. G. Klemens, Phys. Rev. 148, 845 (1966).

${ }^{7}$ A. Bartels, T. Dekorsy, H. Kurz, and K. Kohler, Appl. Phys. Lett. 72, 2844 (1998).

${ }^{8}$ U. Ozugur, C. W. Lee, and H. O. Everitt, Phys. Rev. Lett. 86, 5604 (2001). ${ }^{9}$ R. Merlin, Solid State Commun. 102, 207 (1997).

${ }^{10}$ C. Colvard, T. A. Gant, M. V. Klein, R. Merlin, R. Fischer, H. Morkoc, and A. C. Gossard, Phys. Rev. B 31, 2080 (1985). For a review, see: B. Jusserand and M. Cardona, in Light Scattering in Solids, V, Topics in Appl. Phys. Vol. 66, edited by M. Cardona and G. Guntherodt (Springer, Berlin, 1989).

${ }^{11}$ V. Mitin, V. A. Kochelap, and M. A. Stroscio, Quantum Heterostructures, (Cambridge University Press, Cambridge, 1999).

${ }^{12}$ I. Camps and S. S. Makler, Solid State Commun. 116, 191 (2000).

${ }^{13}$ L. A. Rivlin and A. A. Zadernovsky, Opt. Commun. 100, 322 (1993).

${ }^{14}$ A. R. Bhatt, K. W. Kim, and M. A. Stroscio, J. Appl. Phys. 76, 3905 (1994).

${ }^{15}$ G. Irmer, M. Wenzel, and J. Monecke, Phys. Status Solidi B 195, 85 (1996).

${ }^{16}$ K. Okubo and S. I. Tamura, Phys. Rev. B 28, 4847 (1983). 